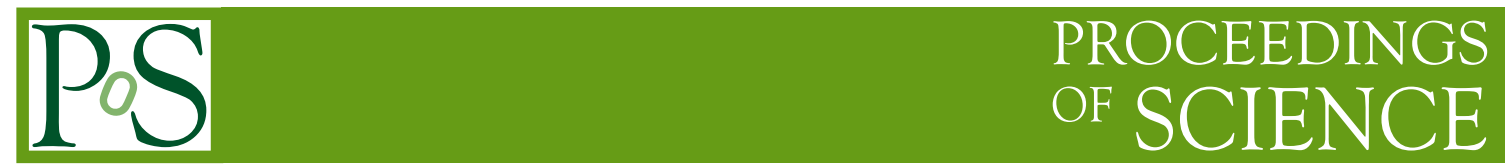

\title{
Social Media strategy for the ATLAS experiment
}

\author{
C. Nellist ${ }^{*+}$
}

LAL-Orsay $(F R)$

E-mail: clara.nellistecern.ch

on behalf of the ATLAS Collaboration.

The ATLAS collaboration uses various social media platforms primarily as a method to communicate the research and achievements of the collaboration to a wider public audience. The strategy to achieve this goal is presented, with an analysis of the effectiveness as a function of certain factors. A specific focus on the social media approach during the LHC Run II time period in 2015 is explored.

38th International Conference on High Energy Physics

3-10 August 2016

Chicago, USA

\footnotetext{
* Speaker.

${ }^{\dagger}$ We acknowledge and thank the contributions of the entire ATLAS collaboration for providing us with ideas and content to share with the world.
} 


\section{Introduction}

The ATLAS collaboration [1][2] uses various social media platforms primarily as a method to communicate the research and achievements of the collaboration to a wider public audience. Broadly, our audience includes students (potential future scientists), the general public, and policy makers. Each group has different reasons to engage which can include: to be informed and to learn, to feel the "wow factor" of big science, and to justify public funding. Users often find our platforms through exposure to, discussion about and interaction with ATLAS content via our initial fan base, as illustrated in fig. 1.

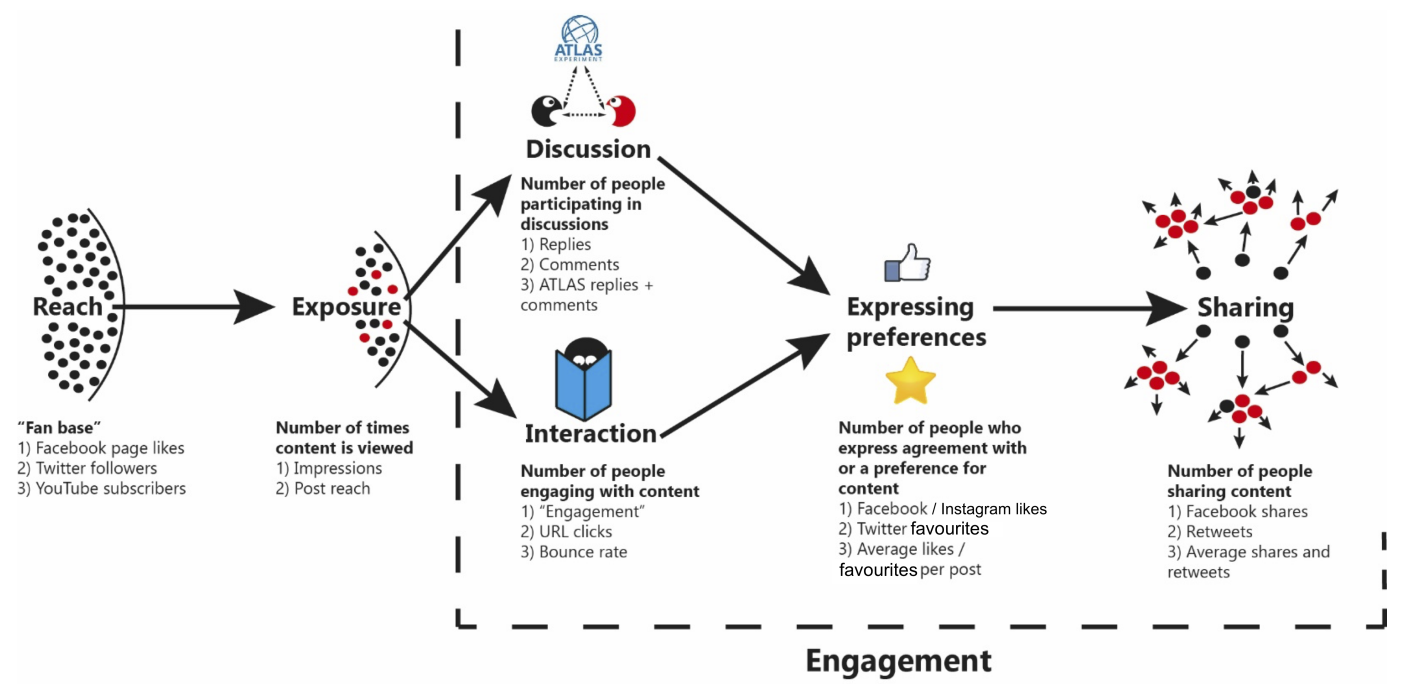

Figure 1: Diagram showing how content can be shared through Social Media platforms [3].

Social media is an important source of information for a large proportion of society now. The aims of the ATLAS social media strategy are:

- To show not just what science is being done, but also how it is done.

- To make ATLAS accessible to a wider audience.

- To share information with other physicists on ATLAS research.

- To reach future ATLAS students and researchers.

- To direct people to the ATLAS website [4] where content is available in more depth. Social Media currently drives around $10 \%$ of our web site referrals. Of that, 59\% Facebook, 24\% Twitter, $10 \%$ Google, $7 \%$ others.

\section{Platforms}

Presented in this Section are the social media platforms used officially by the ATLAS Collaboration. Numbers quoted were up-to-date as of the 29th July 2016.

\subsection{Twitter}

A very popular social media platform with more than 310 million monthly users according to Twitter themselves. Messages (tweets) are posted on the site with a limit of 140 characters. This 
platform is particularly good for live events (called live-tweeting) and discussing popular topics (trending hashtags). Photographs and GIFs are popular and can increase the number of times a tweet is viewed, liked or re-tweeted. Hashtags are useful to tag tweets for a specific topic, but an internal study showed that too many reduce the impact. In-built analytics on the platform allows for the monitoring of audience and of tweet effectiveness [5]. The percentage of ATLAS followers who self-identify as 'male' or 'female' on Twitter is $81 \%$ and $19 \%$ respectively. The ATLAS Twitter account has 37,998 followers and the handle is @ATLASexperiment.

\subsection{Facebook}

Facebook is primarily used to connect people to their family and friends. However, 'Pages' allow people to follow a business, celebrity, or their favourite science experiment. Text in the posts can be longer than on Twitter, allowing more detailed descriptions, and content such as photos (including $360^{\circ}$, shown in fig. 2a) and videos can be embedded. Hashtags were also introduced into Facebook to link common topics. A higher percentage of people following the Facebook page identify as female (27\%) than on Twitter. The page name is @ ATLASexperiment and it has 19,002 likes.

\subsection{Instagram}

An excellent social media platform for ATLAS outreach as it allows the accumulated images on CDS over the past years of ATLAS design, construction, and operation, as well as photographs taken by ATLAS collaborators during their time at CERN to be showcased. For engagement, the focus is on images rather than words, highlighting the aesthetic beauty of scientific design and discovery, creating a good entry point for people of all backgrounds. Additionally, by showing images of events at CERN, ATLAS collaborators, and individuals' photos, we are able to convey the human side of ATLAS research and showcase the multidimensional lives and work of everyone in the collaboration. An example post is shown in fig. 2b. The username is @ ATLASexperiment. This is a relatively new platform for ATLAS, first used in June 2015, and currently has 1,265 followers, so increasing followers and visibility is on-going.

\subsection{Google+}

A social media platform which links automatically to the user's Google account. The posts on the ATLAS G+ account contain the same content as posted onto Facebook. The demographics for the first five countries, as determined by number of users following the ATLAS account, can be seen in reference [5]. It is not known why there is a higher percentage of users identified as female following the account from the United States than in other countries. The account has 122,959 followers and 6,837,402 views. The handle is +ATLASExperiment.

\subsection{Youtube}

YouTube is a video-sharing platform with a highly active user base and the ability to embed content in to other platforms. This makes it an ideal place to host ATLAS video content. A screenshot of an example video is shown in fig. 2c. This is an old platform that has been revived recently. There are 4,087 subscribers to the ATLAS YouTube channel and there have been 1,103,298 views over all videos. The channel name is TheATLASExperiment. 


\section{The ATLAS Social Media Strategy for Run 2 (2015)}

The restart of the LHC for Run 2 in 2015 and the accumulation of data created a rich variety of ATLAS content to share with the public on our platforms. Efforts to increase the number of images and videos were made. Event displays, fig. 2d, were very popular online, and quotes combined with images, example in fig. 2e, increased the likelihood they would be shared and viewed, whilst also overcoming the character limit on Twitter.

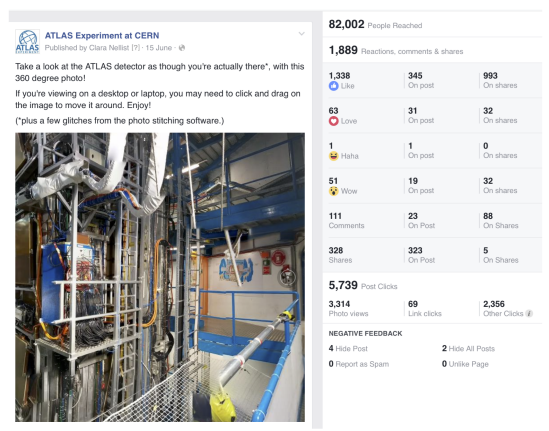

(a)

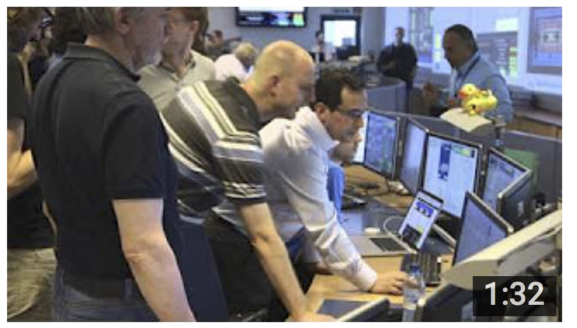

ATLAS in Collision Mode

24,575 views $\cdot 1$ year ago

(c)

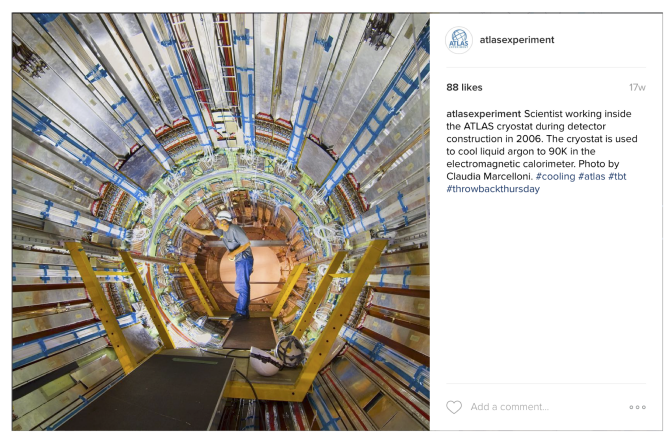

(b)

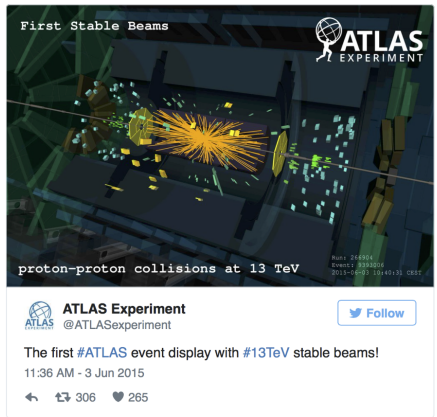

(d)

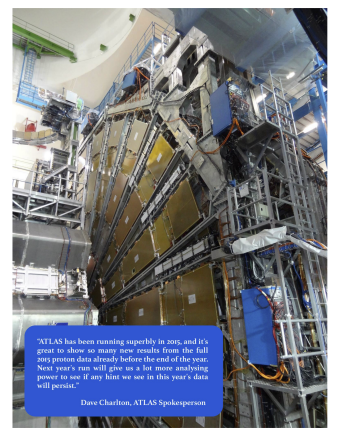

(e)

Figure 2: (a) Post made on Facebook showing a $360^{\circ}$ photo. (b) An example Instagram post on the ATLAS channel. (c) An ATLAS video on YouTube. (d) A tweet with an ATLAS event display shared after the first stable beams at $13 \mathrm{TeV}$. (e) An image with a quote that was shared widely on ATLAS platforms after the End Of Year Event in December 2015.

\section{References}

[1] ATLAS Collaboration, The ATLAS Experiment at the CERN Large Hadron Collider, JINST 3435 (2008) S08003

[2] S. Goldfarb, et. al., Innovating science communication, ATL-OREACH-PROC-2015-003 (2015), https://cds.cern.ch/record/1984340

[3] James Strachan, A Social Media strategy for ATLAS, Master's Thesis (2015).

[4] The ATLAS Collaboration Public Website, http://atlas.cern

[5] C. Nellist, et. al., Social Media strategy for the ATLAS experiment, ATL-OREACH-SLIDE-2016-439 (2016), https://cds.cern. ch/record/2202846 